\title{
HST and ground-based observations of the gravitational lens system Q1009-0252 A \& B ${ }^{\star}$
}

\author{
J.-F. Claeskens ${ }^{1, \star \star}$, S. V. Khmil ${ }^{1,2}$, Dong Wook Lee ${ }^{1}$, D. Sluse ${ }^{1}$, and J. Surdej ${ }^{1, \star \star \star}$ \\ 1 Institut d'Astrophysique et de Géophysique, Université de Liège, Avenue de Cointe 5, 4000 Liège, Belgium \\ 2 Astronomical Observatory of Kyiv University, Observatorna St. 3, UKR-04053 Kyiv, Ukraine
}

Received 20 October 2000 / Accepted 12 December 2000

\begin{abstract}
In this paper, we propose a critical study of direct images and spectra obtained for the doubly imaged QSO Q1009-0252 A \& B (=LBQS1009-0252 A \& B). First, we report new observations with the WFPC2 onboard the Hubble Space Telescope (HST), in which the lensing galaxy is very clearly detected. We combine these with existing NICMOS observations in order to characterize the nature and redshift of the lens. Our HST observations are also fitted with simple analytical lens models; estimates expected for the time delay and M/L of the lensing galaxy are derived. Secondly, new and existing ground-based observations are used in order to establish the first multicolour lightcurves of the lensed components. Although the time resolution is low, flux variability is detected for both components. Finally, ground-based spectroscopic observations published by Surdej et al. (1993) are re-processed with a new method designed to extract spectra with small angular separations. The final spectra are analysed on the basis of a simple model including macrolensing and microlensing amplifications and dust extinction.
\end{abstract}

Key words. quasars: general - gravitational lensing - quasars: individual: Q1009-0252 A \& B

\section{Introduction}

The quasar Q1009-0252 has been identified to be a gravitational lens candidate by Surdej et al. (1993, hereafter Paper I) during a systematic search for multiply imaged quasars within a sample of Highly Luminous Quasars. Photometry $(B, R, i)$ and medium resolution spectroscopy of this system were obtained in March 1992 with the ESO-MPI $2.2 \mathrm{~m}$ telescope (CCD camera) and the $3.5 \mathrm{~m}$ NTT (EMMI spectrograph), respectively, at the European Southern Observatory (La Silla, Chile). Surdej et al. have found that the redshifts of the two QSO images Q1009-0252 A $\left(m_{\mathrm{R}}=17.6\right) \& \mathrm{~B}\left(m_{\mathrm{R}}=20.0\right)$, separated on the sky by 1.55 arcsec, are identical within the measurement errors $(z=2.74, \Delta z \leq 0.001)$ and that the line profiles of Ly $\alpha+\mathrm{NV}, \mathrm{CIV}$ and CIII] look reasonably similar, although the spectral continuum of image A definitely looks bluer than that of image B. They have proposed that Q1009-0252 A \& B consist of double lensed images of the same quasar, and that the blue excess is

\footnotetext{
Send offprint requests to: J. Surdej,

e-mail: surdej@astro.ulg.ac.be

* Based on observations collected at the European Southern Observatory (La Silla, Chile) and with the Hubble Space Telescope.

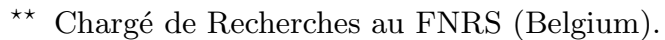

$\star \star \star$ Directeur de Recherches au FNRS (Belgium).
}

possibly induced by some preferential microlensing of image A. An additional point-like component, Q1009-0252 C $\left(m_{\mathrm{R}}=18.9\right)$, has been detected at an angular distance of only $4.6^{\prime \prime}$ from image A. It was found to be a QSO with a redshift $z=1.62$. Absorption due to $\mathrm{MgII}, \mathrm{MgI}$ and numerous FeII lines in the spectra of Q1009-0252 A \& B have been reported at redshifts $z_{\mathrm{a}}=0.866$ and $z_{\mathrm{a}}=1.622$. The absorption line system at $z_{\mathrm{a}}=1.622$ probably arises in gas clouds associated with a cluster containing the quasar Q1009-0252 C. The absorption lines at $z_{\mathrm{a}}=0.866$ were not seen in the spectrum of the latter quasar.

Hewett et al. (1994) have carried out an independent observational study of Q1009-0252 A-C and their results are in good agreement with those of Surdej et al. (1993), with the possible exception of a faint emission feature at $6550 \AA$, which is seen in the spectrum of component B. It is also possibly present, although much fainter, in the spectrum of component A. They have attributed this feature to the redshifted [NIII] $\lambda 1750 \AA$ emission line. Hewett et al. have extensively discussed the two possibilities that Q1009-0252 A \& B are lensed QSO images or constitute a binary quasar.

In Sect. 2, we present direct imaging observations of Q1009-0252 A \& B, obtained with the Hubble Space Telescope and ground-based telescopes. The former allow us to perform precise relative astrometry of the lensed 
images and to derive the photometry and shape of the lensing galaxy, while the latter are used together with data already published in the literature to construct multicolour photometric lightcurves of the lensed QSO images. Section 3 is devoted to the fit of simple lens models reproducing the observations and the mass-to-light ratio of the lens is derived. In Sect. 4, spectroscopic results based on an improved processing of the observational data obtained in Paper I are reported and analysed in terms of extinction by dust and microlensing in the lensing galaxy. Finally, our conclusions are summarized in Sect. 5.

Except when stated otherwise, we adopt in this paper a flat cosmological model dominated by a cosmological constant, with parameters: $\Omega_{\mathrm{o}}=0.3, \lambda_{\mathrm{o}}=0.7$ and $H_{\mathrm{o}}=$ $65 \mathrm{~km} \mathrm{~s}^{-1} / \mathrm{Mpc}$.

\section{Direct imaging of Q1009-0252 A \& B}

In this section, we first describe and analyse observations of Q1009-0252 A \& B obtained with the Hubble Space Telescope (HST); then we review existing and present new ground-based imaging of this multiply imaged quasar with the aim of constructing the lightcurves of the different lensed images.

\subsection{HST direct imaging}

HST images of Q1009-0252 were obtained with the WFPC2 planetary camera on January 1, 1999 (HST proposal ID 6790). They consist of 4 dithered exposures of $400 \mathrm{~s}$ with the F555W (nearly Johnson $V$ ) filter and 2 exposures of $1300 \mathrm{~s}$ with the F814W (nearly Kron-Cousins $\left.I_{\mathrm{c}}\right)$ filter. The CCD scale is $0.0455^{\prime \prime} /$ pixel and a gain of $15 \mathrm{e}^{-} /$ADU was chosen to avoid the saturation of the bright QSO components.

The lensing galaxy $\mathrm{G}$ is only detected on the $\mathrm{F} 814 \mathrm{~W}$ frames, as a diffuse object located between the A and B QSO components (see left panel in Fig. 1). The lens has also been independently discovered by the CASTLE program on NICMOS frames in the $H$ band (Lehár et al. 2000). The host galaxy of quasar $\mathrm{C}$ and a faint galaxy close to component A, which are also detected with NICMOS, are not visible on our frames.

\subsubsection{PSF analysis}

Astrometry and photometry of the lensed QSO components have been derived from the simultaneous fit of the positions and intensities of two Point Spread Functions (PSFs). Synthetic PSFs have been generated with the TINYTIM software package (Krist \& Hook 1996). The analysis has been carried out as described in Remy et al. (1998). Since the host galaxy of QSO C remained undetected, this quasar proved to be an appropriate point-like object in the field and has been used to generate for each frame an oversampled TINYTIM PSF for the optimal values of the jitter and focus parameters. Then, an iterative procedure addressed the problem of fitting the HST PSF
Table 1. Relative astrometry and photometry of Q1009-0252 $\mathrm{A}, \mathrm{B} \& \mathrm{C}$ and of the lensing galaxy $\mathrm{G}$ in the $V$ and $I_{\mathrm{c}}$ bands. The error bars on the photometry of the point-like images and on the astrometry are formal errors resulting from the fit. The photometric error on the galaxy mainly results from uncertainties in the sky subtraction

\begin{tabular}{lrrcc}
\hline \hline & $\Delta \alpha \cos \delta\left(^{\prime \prime}\right)$ & $\Delta \delta\left(^{\prime \prime}\right)$ & $V$ & $I_{\mathrm{c}}$ \\
\hline $\mathrm{A}$ & $0.000 \pm 0.000$ & $0.000 \pm 0.000$ & $18.51 \pm 0.01$ & $17.92 \pm 0.01$ \\
$\mathrm{~B}$ & $-0.671 \pm 0.001$ & $-1.382 \pm 0.001$ & $21.04 \pm 0.01$ & $19.98 \pm 0.01$ \\
$\mathrm{C}$ & $-4.294 \pm 0.001$ & $1.627 \pm 0.001$ & $19.15 \pm 0.01$ & $18.71 \pm 0.01$ \\
$\mathrm{G}$ & $-0.538 \pm 0.005$ & $-1.092 \pm 0.005$ & $>23.8$ & $21.76 \pm 0.30$ \\
\hline
\end{tabular}

peak after proper re-centering and re-sampling of the final TINYTIM PSF. The central panel of Fig. 1 shows the residuals after subtraction of two scaled PSFs corresponding to the A and B QSO images. Let us note that the bright residuals seen in the vicinity of the A position are due to an artefact caused by the imperfect TINYTIM PSF. Indeed, their integrated flux is less than $2 \%$ of the total flux in the PSF.

On the F814W frames, a two-dimensional analytical model has been fitted to the lensing galaxy simultaneously with the numerical PSFs. A pure exponential model (Freeman 1970) and a pure de Vaucouleurs model (de Vaucouleurs 1948) have been individually considered. In both cases, the relative position of the galaxy is the same and is found to be located very close to the line joining the $\mathrm{A} \& \mathrm{~B}$ lensed images. The angular separation between $\mathrm{A}$ and $\mathrm{G}$ is derived to be $\Delta \theta_{\mathrm{AG}}=1.217 \pm 0.007^{\prime \prime}$ while $\Delta \theta_{\mathrm{AB}}=1.536 \pm 0.001^{\prime \prime}$, in agreement with Lehár et al. (2000). The relative astrometry of G and Q1009$0252 \mathrm{~A}, \mathrm{~B} \& \mathrm{C}$ is reported in the first columns of Table 1.

The photometry of the point-like components has been derived from the integrated number counts of the corresponding scaled PSFs, within a radius of 3 arcsec (Remy et al. 1997). On the other hand, the magnitude of the galaxy has been estimated from aperture photometry (within $0.5^{\prime \prime}$ from the galaxy center) on the residuals left after subtraction of the QSO components. This approach is nearly insensitive to the galaxy modeling. Then, the number counts have been transformed into Johnson $V$ and Kron-Cousins $I_{\mathrm{c}}$ magnitudes using the PHOTFLAM keyword present in the frame headers, applying CTE corrections and zero point corrections from STMag to Vega systems, as described in Whitmore (1997). For the lens, a further colour correction $\mathrm{F} 814 \mathrm{~W}-I_{\mathrm{C}} \simeq 0.1$ mag has been added (Fioc \& Rocca-Volmerange 1997; Fukugita et al. 1995). The photometric results are listed in the last two columns of Table 1. The lower limit of the $V$ magnitude of the lensing galaxy has been estimated by analysing the real image after addition of synthetic galactic profiles with various magnitudes. 


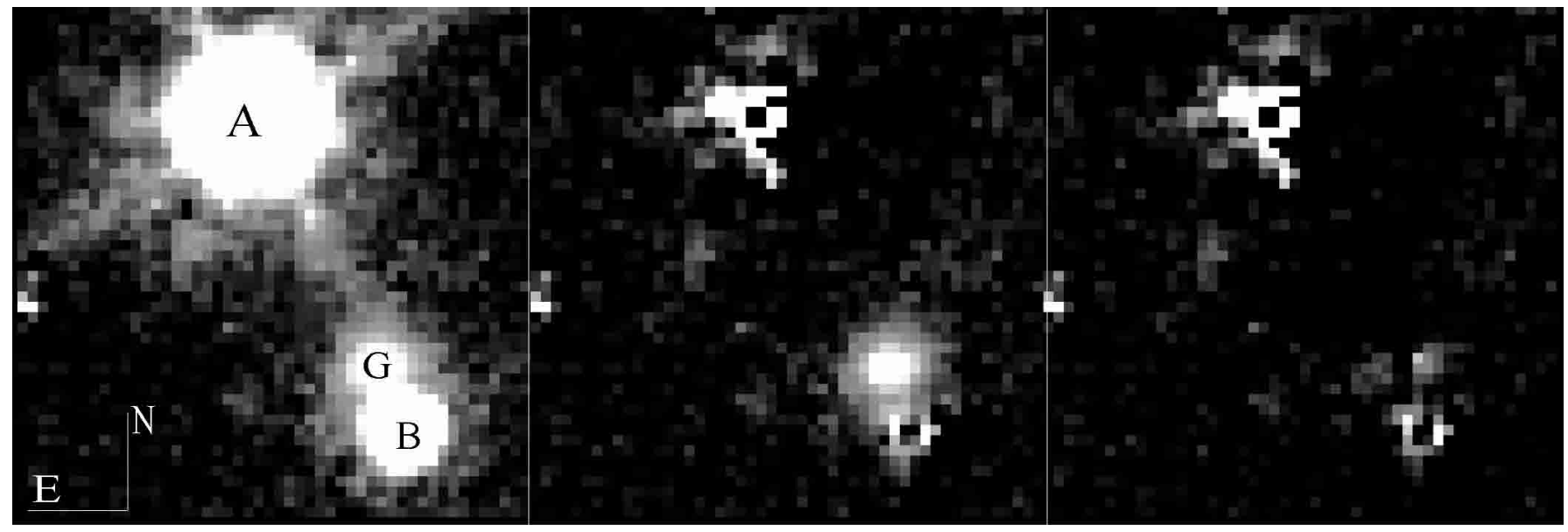

Fig. 1. Direct imaging with the planetary camera onboard HST of Q1009-0252 A \& B and the lensing galaxy, in the F814W spectral band: coadded frames (left), residuals after subtraction of the quasar images with scaled PSFs (middle) and after a further subtraction of the lensing Galaxy G with a de Vaucouleurs model (right). North is up and East to the left. The angular separation between $\mathrm{A}$ and $\mathrm{B}$ is $1.54^{\prime \prime}$

Table 2. Shape parameters of the lensing galaxy for the exponential (Exp) and de Vaucouleurs (dVc) intensity profiles. The angular scale length $r_{\mathrm{s}}$ represents the characteristic length $r_{\mathrm{c}}$ (resp. the effective radius $r_{\mathrm{e}}$ ) of the Exp (resp. dVc) galactic profile. The error bars represent half the difference between the results obtained from the two individual frames

\begin{tabular}{lcc}
\hline \hline & $\operatorname{Exp}$ & $\mathrm{dVc}$ \\
\hline Axis ratio & $0.65 \pm 0.01$ & $0.60 \pm 0.04$ \\
Position angle $(\mathrm{N} \rightarrow \mathrm{E})$ & $10^{\circ} .5 \pm 3^{\circ}$ & $9^{\circ} .6 \pm 3^{\circ}$ \\
Scale length $r_{\mathrm{s}}\left(^{\prime \prime}\right)$ & $0.109 \pm 0.005$ & $0.571 \pm 0.062$ \\
\hline
\end{tabular}

\subsubsection{The lensing galaxy}

In order to characterize the morphology of the lensing galaxy, the latter has been fitted with convolved Exponential (Exp) and de Vaucouleurs (dVc) intensity profiles. Unfortunately, it is impossible to state which one is the best model from a simple comparison between their residuals or their $\chi^{2}$ minima, which are equivalently good. The shape parameters of the models are reported in Table 2.

The fitted value of the effective radius of the $\mathrm{dVc}$ model listed in Table 2 is quite different from the value $r_{\mathrm{e}}=0.19^{\prime \prime} \pm 0.04^{\prime \prime}$ obtained by Lehár et al. (2000) on the basis of $H$-band imagery. We have checked the significance of our result by means of simulations. First, we have built synthetic observations with either an Exp or a dVc lens intensity profile. We have chosen the parameters in accordance with the values listed in Tables 1 and 2 and the signal to noise ratio $\mathrm{S} / \mathrm{N}$ to be the same as in our data, but a random intra-pixel centering of the lens model is operated. We have then applied the same analysis as for the real observations. The scale length has been found to be equal to the input value for the Exp model and could be underestimated at most by $15 \%$ for the dVc model, depending on the exact centering. This proved the stability of the algorithm. Second, a synthetic observation has been generated, with a dVc profile for the lens with $r_{\mathrm{e}}=0.19^{\prime \prime}$ and the $\mathrm{S} / \mathrm{N}$ present in our data. Then, it has been fitted with a $\mathrm{dVc}$ profile whose effective radius was fixed to the value $r_{\mathrm{e}}=0.57^{\prime \prime}$. The residuals left after the subtraction of the model are not compatible with those obtained on the real images.

Although our derived value of the effective radius is quite normal for a high redshift elliptical galaxy (it corresponds to about $4.8 h_{65}^{-1} \mathrm{kpc}$ for $z_{l} \simeq 1$ ), the expected decrease of $r_{\mathrm{e}}$ at longer wavelengths as shown by Pahre et al. (1998) is not sufficient to explain the large difference between our estimate and that derived by Lehár et al. (2000) in the $H$-band.

As we have already mentioned, the morphological type of the lensing galaxy cannot be determined on the sole basis of profile fitting. Simulations with the $\mathrm{S} / \mathrm{N}$ present in our data indicate that the lens has not a pure $\mathrm{dVc}$ profile with $r_{\mathrm{e}} \simeq 0.6^{\prime \prime}$, since then, the fit of an Exp model would leave significant residuals. Thus, the lens intensity profile could result from a combination of the bulge and the disk (e.g. Schmidtke et al. 1997), but the $\mathrm{S} / \mathrm{N}$ is too low to fit such a composite profile. However, the photometry of the lens provides us with additional informations. Figure 2 shows the $V-I_{\mathrm{c}}$ and $I_{\mathrm{c}}-H$ colours of elliptical and spiral galaxies as a function of their redshift, as predicted by the Pegase galaxy evolution model developed by Fioc \& Rocca-Volmerange (1997). The expected colours of the galaxies do not much depend on the adopted cosmological model and the observed colour indices $\left(V-I_{\mathrm{c}}\right)_{\mathrm{obs}}>2.04$ (see Table 1 ) and $\left(I_{\mathrm{c}}-H\right)_{\mathrm{obs}}=2.5 \pm 0.3$ (see Table 1 and Lehár et al. 2000) indicate that the lensing galaxy should preferentially be an $L \simeq 1.14 L_{*}$ elliptical at $z_{1} \simeq$ $0.8 \pm 0.2$. Thus, the latter could possibly be associated with the system of narrow metal absorption lines observed in the spectra of both A \& B components at $z_{\mathrm{a}} \simeq 0.87$ (Paper I, Hewett et al. 1994 and Sect. 4.3). The mass-to-light 

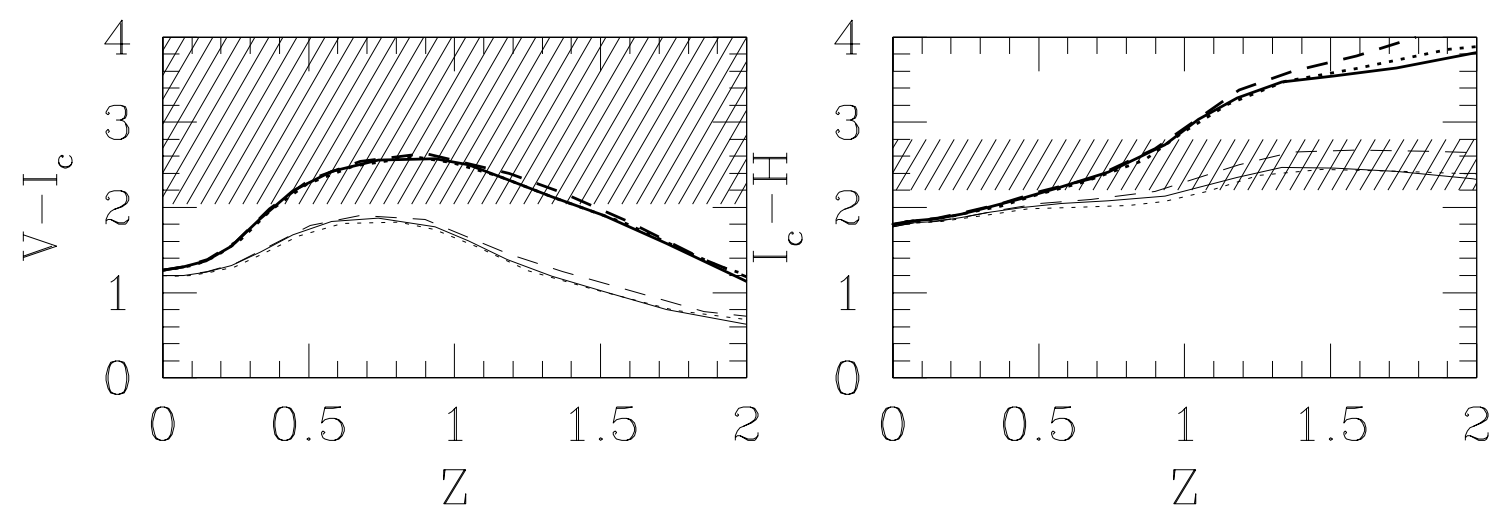

Fig. 2. Expected $V-I_{\mathrm{c}}$ (left) and $I_{\mathrm{c}}-H$ (right) colour indices for elliptical (thick lines) and spiral galaxies (thin lines) and for different cosmologies: $\left(\Omega_{\mathrm{o}}, \lambda_{\mathrm{o}}, h_{100}\right)=(0.3,0.7,0.65)$ (full lines), $(0.1,0,0.65)$ (dashed lines) and $(1,0,0.5)($ dotted lines). The hatched regions correspond to the values of the colour indices permitted by the HST observations

Table 3. Summary of the ground based direct imaging observations of Q1009-025 A \& B.

\begin{tabular}{lcccc}
\hline \hline Telescope & Date & Filter & Seeing $\left({ }^{\prime \prime}\right)$ & Ref. \\
\hline ESO/MPI 2.2 m & $24-25 / 02 / 92$ & $B$ & 0.91 & (a) \\
ESO/MPI 2.2 m & $24-25 / 02 / 92$ & $R$ & 0.75 & (a) \\
ESO/MPI 2.2 m & $25-26 / 02 / 92$ & $i$ & 1.2 & (a) \\
JKT 0.9 m & $01-02 / 04 / 92$ & $B$ & 1.1 & (b) \\
JKT 0.9 m & $01-02 / 04 / 92$ & $V$ & 0.9 & (b) \\
JKT 0.9 m & $01-04 / 11 / 92$ & $B$ & 1.1 & (c) \\
JKT 0.9 m & $01-04 / 11 / 92$ & $V$ & 0.9 & (c) \\
JKT 0.9 m & $01-04 / 11 / 92$ & $r$ & 0.8 & (c) \\
JKT 0.9 m & $01-04 / 11 / 92$ & $i$ & 0.8 & (c) \\
ESO/MPI 2.2 m & $20-21 / 03 / 93$ & $B$ & 1.04 & (d) \\
ESO/MPI 2.2 m & $21-22 / 03 / 93$ & $R$ & 0.75 & (d) \\
ESO/MPI 2.2 m & $21-22 / 03 / 93$ & $i$ & 0.68 & (d) \\
NTT & $11-12 / 02 / 94$ & $B$ & 1.16 & (d) \\
NTT & $11-12 / 02 / 94$ & $R$ & 0.88 & (d) \\
NTT & $11-12 / 02 / 94$ & $i$ & 0.91 & (d) \\
\hline
\end{tabular}

(a): Surdej et al. (1993); (b): Hewett et al. (1994); (c): Hewett et al. (1994), non photometric conditions; (d): Claeskens et al., this paper.

ratio derived in Sect. 3.1 is also more similar to that of an early-type galaxy.

\subsection{Ground-based direct imaging}

The photometry of Q1009-025 A \& B performed with HST in 1999 and listed in Table 1 indicates, in comparison with the observations reported by Hewett et al. (1994), that since 1992 both lensed components have faded by about $0.5 \mathrm{mag}$ in both the $V$ and $I_{\mathrm{c}}$ bands. This has prompted us to build photometric lightcurves from our unreleased ground-based observations as well as from the results published by Surdej et al. (1993) and Hewett et al. (1994) (see Table 3 for a summary).

\subsubsection{New observations}

After its discovery reported in Paper I, Q1009-025 A \& B has been imaged in the $B, R$ and $i$ bands at the European Southern Observatory (ESO, La Silla, Chile) in March 1993 with a direct CCD camera mounted at the Cassegrain focus of the ESO/MPI $2.2 \mathrm{~m}$ telescope, and in February 1994 with the SUSI direct camera mounted at the Nasmyth focus of the New Technology Telescope (NTT). The scale on the sky was $0.175^{\prime \prime} /$ pixel and $0.128^{\prime \prime} /$ pixel, respectively. Photometric conditions prevailed during the observations and standard fields (Landolt 1992) were acquired each night for flux calibrations. Despite the good seeing conditions, the lensing galaxy remained undetected even in the $i$ band.

Once the standard image reduction has been performed, a numerical PSF is constructed for each frame from isolated stars present in the field. The simultaneous fit of the peak intensity and position of two PSFs on Q1009-0252 A \& B leaves absolutely no residuals. The photometry of both QSO lensed images is then obtained from the integrated number counts in the respective scaled PSFs. Simulations show that the stochastic errors on the individual magnitudes of components $\mathrm{A}$ and $\mathrm{B}$ are at most 0.01 and $0.03 \mathrm{mag}$, respectively. The systematic error on the zero point is estimated to be $0.03 \mathrm{mag}$.

\subsubsection{Multicolour photometric lightcurves}

Figure 3 illustrates the $B, V, R$ and $i$ lightcurves of Q10090252 A \& B as well as their corresponding differential lightcurves, obtained between February 1992 and January 1999. They have been derived from all the ground-based observations listed in Table 3 and from the WFPC2 photometry presented in Sect. 2.1. The lightcurves of Q1009-025 C are not illustrated but they show no sign of photometric variability. Assuming that QSO C is photometrically stable, we calibrated the non photometric part of the data obtained by Hewett et al. (1994). Moreover, a correction of 0.05 mag (Fukugita et al. 1995) has been 

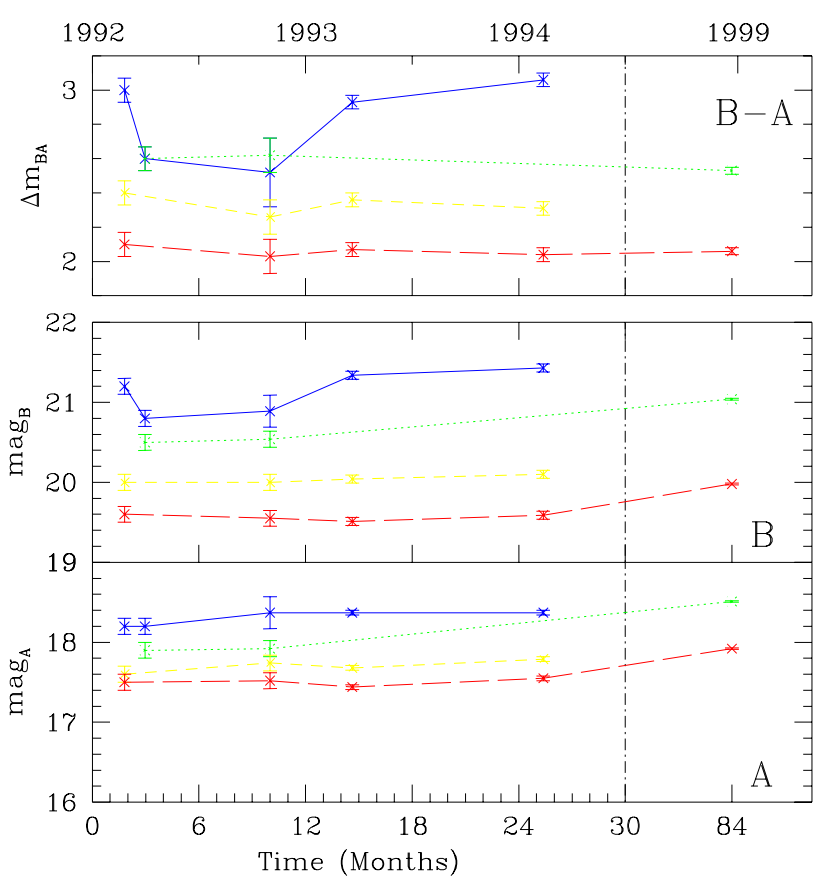

Fig. 3. Lightcurves between 1992 and 1999 for Q1009-0252 A (bottom panel) and B (middle panel), in the $B$ (full lines), $V$ (dotted lines), $R$ (short dashed lines) and $i$ (long dashed lines) bands. The top panel shows the corresponding B-A differential lightcurves

applied to their Gunn-r magnitudes to convert these to the Johnson- $R$ band.

Considering first the differential lightcurves in Fig. 3, it is clear that the magnitude difference between the lensed images is a decreasing function of wavelength. This could be the signature of dust-extinction affecting the $B$ image, which is located close to the lensing galaxy (see Sect. 4.3). It is also noticeable that the magnitude difference in the $B$ band has significantly decreased between February 1992 and March 1993 (Hewett et al. data). Looking now at the absolute lightcurves in Fig. 3, it appears that the $B$ component has brightened in the $B$ filter during the same period, while the A component was getting slightly fainter. The other lightcurves remained more or less constant. This could be due to an intrinsic change in the blue part of the QSO spectrum during that period. The very poor time sampling does not allow to establish a time delay; conversely the expected time delay of about 120 days (see Sect. 3.2) is not in conflict with the observations if the quasar undergoes fast flux variations. Of course, microlensing could also affect the blue part of the spectrum of image $B$ and superpose itself to fainter intrinsic flux variations... However, an additional proof of intrinsic flux variations is given by the dimming of both lensed components as detected by HST in the $V$ and $I_{\mathrm{c}}$ bands in early 1999. These intrinsic flux variations should draw our attention on the interest of monitoring this doubly imaged quasar in order to determine the time delay and better constrain the lens model or estimate the Hubble constant.

\section{Lens model and lens properties}

\subsection{Lens model fitting}

Close examination of the HST astrometry (see Table 1) shows that the lens is not perfectly aligned with the lensed QSO images, so that a deviation from circular symmetry must be included into the lensing potential in order to reproduce the lens configuration. The easiest way to proceed is to model the lens with a Singular Isothermal Sphere (SIS) and to add a constant external shear $\gamma$ acting as a perturbation. The lens equation can be written as follows (e.g. Schneider et al. 1992):

$\boldsymbol{\eta}=\boldsymbol{\xi}-\Gamma \boldsymbol{\xi}-\theta_{\mathrm{E}}\left(\frac{\boldsymbol{\xi}}{|\boldsymbol{\xi}|}\right)$

where

$\Gamma=\gamma\left(\begin{array}{rr}\cos 2 \varphi & \sin 2 \varphi \\ \sin 2 \varphi & -\cos 2 \varphi\end{array}\right)$

and

$\theta_{\mathrm{E}}=\frac{4 \pi \sigma_{v}^{2}}{c^{2}}\left(\frac{D_{\mathrm{ls}}}{D_{\mathrm{os}}}\right)$

are the external shear tensor (with module $\gamma$ and orientation $\varphi$ ) and the SIS angular Einstein radius, respectively ( $D_{\mathrm{ls}}$ and $D_{\mathrm{os}}$ are cosmological angular distances). $\boldsymbol{\eta}$ and $\boldsymbol{\xi}$ in Eq. (1a) are the two-dimensional angular vectors in the source plane and in the lens plane, respectively. Equation (1a) can be inverted to find the image positions (see Appendix). The model parameters are then found by means of $\chi^{2}$ minimizations, which are performed with the downhill simplex method (Press et al. 1990).

Adopting the astrometric results presented in Table 1 and the magnitude difference in the $H$ band between the lensed images $\Delta H=1.57$ (i.e. a flux ratio of 4.25 , Lehár et al. 2000) in order to minimize the effects of extinction, we have derived the model parameters listed in the first row of Table 4 . The corresponding source and image configurations are illustrated in Fig. 4. The resulting orientation of the shear does not lead to the identification of an associated object.

The parameter values listed in Table 4 are slightly sensitive to the adopted flux ratio between the lensed images. If the intrinsic flux ratio $F_{\mathrm{i}}$ is smaller than observed in the $H$ band, the shear also gets smaller. However, if $F_{\mathrm{i}}$ is smaller than the flux ratio expected with the pure SIS model, i.e. $F_{\mathrm{i}} \lesssim 3.8$, the expected shear increases again, but with an orientation $\varphi \simeq 120 \mathrm{deg}$ which is then compatible with that of the shear produced by Q1009-025 C. Let us note here that the 5 observational constraints given by the two lensed images are not sufficient to get a unique solution when including Q1009-025 C and/or its host galaxy in the lens model.

Of course, the asymmetry of the lensing potential may also be due to an intrinsic ellipticity $\epsilon$ of the matter distribution. We have also fitted the Singular Isothermal Ellipsoid (SIE) developed by Kormann et al. (1994) and 
Table 4. The best fit parameters for the SIS plus shear and SIE lens models. The source position $\left(\eta_{1}, \eta_{2}\right)$ is relative to that of the lens

\begin{tabular}{lccccc}
\hline Model & $\theta_{\mathrm{E}}\left({ }^{\prime \prime}\right)$ & $\gamma / / \epsilon$ & $\varphi$ (deg.) & $\begin{array}{c}\eta_{1}\left({ }^{\prime \prime}\right) \\
(\text { East })\end{array}$ & $\begin{array}{c}\eta_{2}\left({ }^{\prime \prime}\right) \\
(\text { North })\end{array}$ \\
\hline \hline SIS $+\gamma$ & $0.780 \pm 0.005$ & $0.017 \pm 0.009$ & $11.7 \pm 16$ & $0.192 \pm 0.003$ & $0.413 \pm 0.003$ \\
SIE & $0.774 \pm 0.005$ & $0.051 \pm 0.024$ & $11.8 \pm 16$ & $0.193 \pm 0.003$ & $0.406 \pm 0.003$ \\
\hline
\end{tabular}

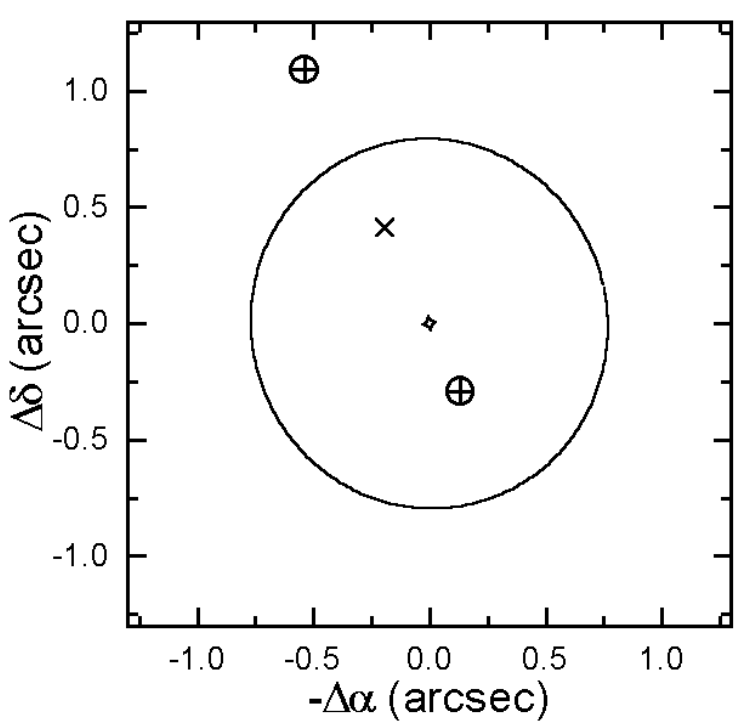

Fig. 4. Configuration of Q1009-025 A \& B fitted with the SIS + shear lensing model. The "+" symbols represent the fitted image positions and the "O" symbols represent the observed positions. The derived source position is denoted by a " $\times$ ". The large circle represents the critical line in the lens plane, and the small diamond shape is the corresponding caustic in the source plane. All positions are projected on the sky

Kassiola \& Kovner (1993). The observations described above are reproduced by this lens model if the ellipticity $\epsilon=1-f$, where $f$ is the axis ratio, is equal to 0.051 (see second row of Table 4). Finally, in both lens models, the total amplification of the QSO is 1.37 mag.

\subsection{Time delay}

We know from Sect. 2.2.2 that the lensed components show flux variations which might be intrinsic to the source. These flux variations are not simultaneously seen in both lensed images, but they are separated by a time delay (Refsdal 1964). Within the SIE lens model, the time delay separating flux variations in the individual components may be computed from the observed image positions, using the simple exact relation derived by Witt et al. (2000). Assuming the lens redshift is $z_{1}=0.87$, as proposed in Sect. 2.1.2, $\Delta t_{\mathrm{AB}}=120.8 h_{65}^{-1}$ days, with image A leading. Within the SIS $+\gamma$ lens model, a slight increase of the time delay of about $2 \%$ is expected due to the small shear effect. However, $\Delta t_{\mathrm{AB}}=108.6 h_{65}^{-1}$ days is expected if $\Omega_{\mathrm{o}}=1$ and $\lambda_{\mathrm{o}}=0$. Thus, the uncertainty due to the lens model is smaller than that due to the cosmological model, because the assumed lens redshift is rather high.

\subsection{Lens properties}

Assuming that the lensing galaxy is an elliptical at $z_{1}=$ 0.87 , it is easy to derive its velocity dispersion from the value of $\theta_{\mathrm{E}}$ reported in Table 4 and from Eq. (1c): $\sigma_{\mathrm{v}}=230 \mathrm{~km} \mathrm{~s}^{-1}$. Then, according to the Faber-Jackson (1976) relation, the lens is a $L=1.08 L_{*}$ galaxy, in good agreement with the value $L=1.14 L_{*}$ derived from HST photometry in Sect. 2.1.2.

Finally, the mass-to-light ratio of the lens can be derived from our observations in a nearly model-independent way. First, the surface mass density within the angular Einstein radius being equal to the critical density, the deflecting mass within $\theta_{\mathrm{E}}$ is $\mathrm{M}_{\mathrm{E}}=2.510^{11} h_{65}^{-1} M_{\odot}$ (with $z_{\mathrm{s}}=2.74, z_{1}=0.87$ and $\theta_{\mathrm{E}}$ given in Table 4 ). Secondly, the lens aperture photometry within $\theta_{\mathrm{E}}$ yields $m_{I_{\mathrm{c}, \mathrm{E}}}=21.46 \pm 0.1$, corresponding to an absolute magnitude $M_{I \mathrm{c}, \mathrm{E}}=-22.66+5 \log h_{65}$ (Fioc \& RoccaVolmerange 1997). Adopting $M_{I_{\mathrm{c}}, \odot}=4.2$ (Allen 1976 and colour transformation by Cousins 1976), we get $L_{I_{\mathrm{c}, \mathrm{E}}}=$ $5.510^{10} h_{65}^{-2} L_{\odot}$. The mass-to-light ratio in the $I_{\mathrm{c}}$ band is thus $\left(M_{\mathrm{E}} / L_{\mathrm{E}}\right)_{I_{\mathrm{c}}}=4.5 \pm 0.5 h_{65}$ in solar units. Taking into account the expected $\left(B-I_{\mathrm{c}}\right) \simeq 2.2$ colour for the galaxy, this mass-to-light is then converted into the $B$ band: $\left(M_{\mathrm{E}} / L_{\mathrm{E}}\right)_{B}=10.5 \pm 1 h_{65}\left(M_{\odot} / L_{\odot}\right)_{B}$. This value is quite typical of the observed global mass-to-light ratio of local elliptical galaxies (Lauer 1985). It is also typical of other elliptical gravitational lenses (Keeton et al. 1998). Let us note that in the Einstein - de Sitter universe, the derived mass-to-light ratio would be twice larger but still, the galaxy could not be considered as a "dark lens" (Hawkins 1997).

\section{Spectroscopy of Q1009-0252 A \& B}

\subsection{Observations}

Three high quality spectroscopic datasets covering the wavelength range $\lambda \lambda 3900-7600 \AA$ were obtained for Q1009-0252 A \& B in March 1992 with the EMMI spectrograph (red grating \# 13) at the Nasmyth focus of the $3.5 \mathrm{~m}$ NTT. Each of these datasets was exposed during 30 min through a long slit aligned with the $\mathrm{A}$ and $\mathrm{B}$ lensed images; the slitwidth was $1^{\prime \prime}$ and the seeing at the time of the observations was $0.85^{\prime \prime}, 0.85^{\prime \prime}$ and $0.71^{\prime \prime}$, respectively. The scale along the spatial direction is $0.44^{\prime \prime} /$ pixel, corresponding to slightly undersampled spectra along the slit. The effective spectral resolution is approximately $10 \AA$ $(1$ pixel $\simeq 4 \AA)$. 


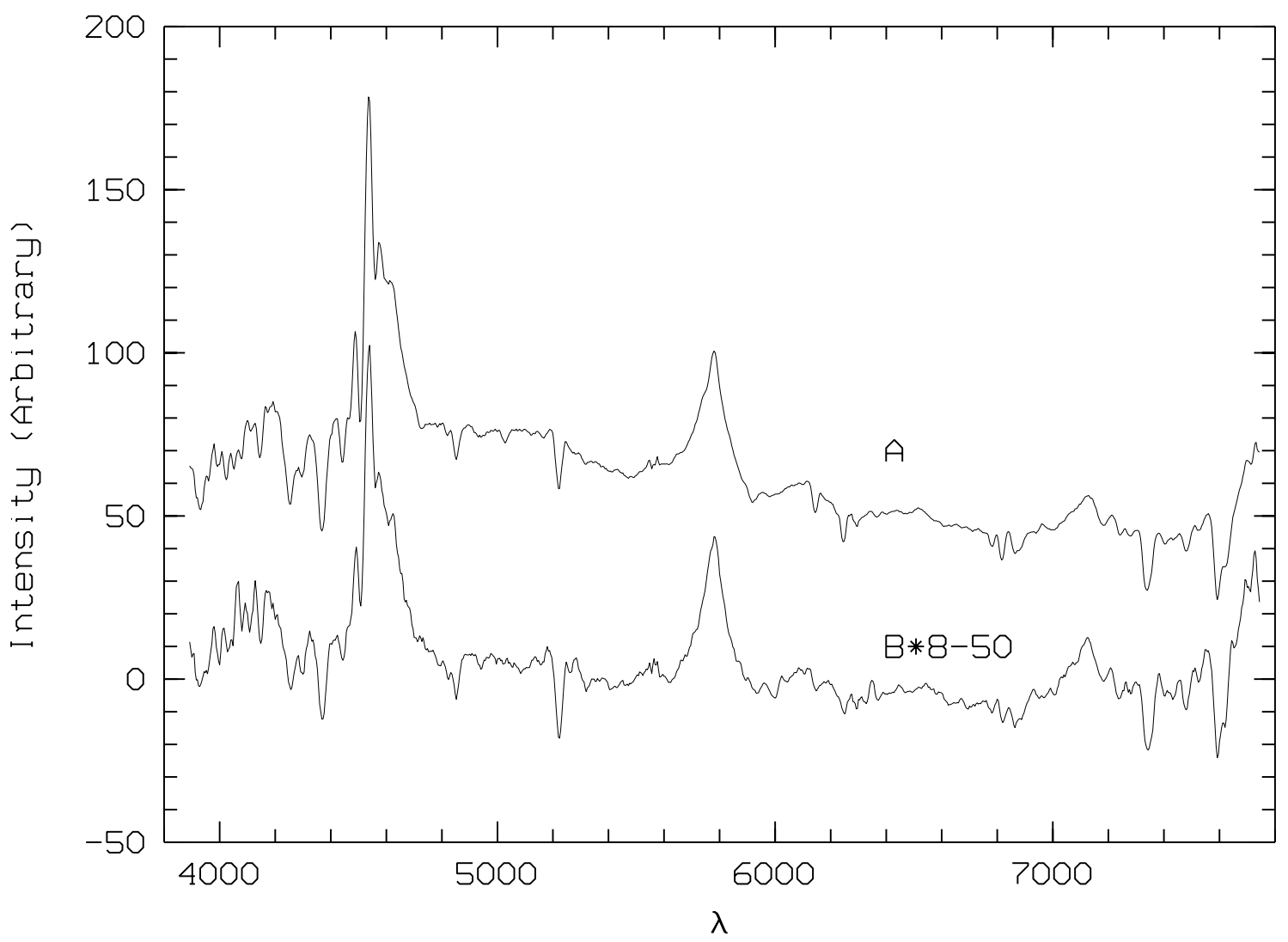

Fig. 5. Spectra of Q1009-0252 A (top) and B (bottom) extracted using the MEM method described in this work. Spectrum of $\mathrm{B}$ has been multipled by a factor 8 and offset by 50 counts. All the data have been slightly smoothed using a 5 pixels filter box

\subsection{Data extraction}

The spectra presented in Paper I were extracted adapting the method developed by Horne (1986) for a single object spectrum to the case of multiple point-like sources. This method performs an optimal extraction by translating along the slit direction a mask constructed from the spectrum of the bright component, taking into account noise due to the CCD read-out (10 $\mathrm{e}^{-}$in this case), the object and the sky. The three masks turn out to be slightly curved along the spectral dispersion due to differential refraction. Assuming that individual spectrum profiles along the slit are symmetrical, we have decontaminated the spectrum of the faint $\mathrm{B}$ component by light due to the bright one, and vice versa. Average spectra extracted in this way have been shown for the A, B and also $\mathrm{C}$ components in Figs. 1 and 2 of Paper I.

In order to check those results based on a somewhat artisanal extraction method, we have decided to re-extract the spectra using a totally independent technique. A general algorithm of spectra extraction for a system of pointlike sources (cf. multiple lensed images of a same quasar) based on the maximum entropy method (MEM) has been developed by Khmil \& Surdej (2000). We only describe here its general features.

It is well known that, when deconvolving spectra of point-like objects, one usually deals with three sets of unknown parameters: the deconvolved spectra themselves (i.e. the emitted flux versus wavelength), the point-spread function induced by the atmosphere and the instrument, and the positions of the spectra relative to the CCD frame; these may vary because of refractive dispersion or when the slit is not perfectly aligned with the columns of the CCD. The main idea of the proposed algorithm is to apply the MEM to each independent set of parameters and to improve their values by successive iterations. The parameter sets obtained at a given stage of iteration serve as the default input to the next stage. The algorithm makes use of all the a priori knowledge available about the spectra (e.g. flux positivity, relative magnitudes between the components if these are known, relative astrometry, seeing FWHM, etc.). The latter actually provide the default values of the initial parameter sets. This algorithm has been extensively tested on simulated data presenting characteristics (noise properties, CCD sampling, ...) similar to the NTT spectroscopic observations. The issue of flux conservation was also very carefully checked and the results look very encouraging (Khmil \& Surdej 2000).

\subsection{Spectroscopic results}

Figure 5 displays the MEM extracted spectra of Q1009$0252 \mathrm{~A} \& \mathrm{~B}$, corresponding to the co-addition of the individual results obtained after processing the three $\mathrm{CCD}$ frames. These final spectra are found to be in very good agreement with those extracted using the artisanal 
extraction method presented in Paper I and we therefore feel very confident that all the similarities and slight differences already discussed and reported there between these spectra are definitely real. In particular, the redshifts derived from the Ly $\alpha+\mathrm{NV}, \mathrm{CIV}$ and CIII] emission lines are respectively found to be 2.739 and $2.740( \pm 0.001)$ for the $\mathrm{A}$ and $\mathrm{B}$ components. We confirm that the line profiles of Ly $\alpha+\mathrm{NV}$, CIV and CIII] look rather similar on the whole. However, some significant differences are conspicuous. For instance, the equivalent widths measured for the Ly $\alpha+\mathrm{NV}$, CIV and CIII] emission lines are found to be 106, 92, $24 \AA$ and 139, 106, $32 \AA$ for the A and B components, respectively. Hewett et al. (1994) have observed the same trend of somewhat larger equivalent widths for the emission lines observed in the spectrum of the B component, with respect to A. Furthermore, the spectrum of Q1009-0252 A definitely looks bluer than that of B, in full agreement with the results presented in Sect. 2.2.2, with those presented in Paper I based upon broad band photometry and spectroscopy, and with the independent work by Hewett et al. (1994). In particular, the faint emission feature reported by Hewett et al. in the spectrum of component B at $6550 \AA$, due to the redshifted [NIII] $\lambda 1750 \AA$ emission line, is faintly visible in the spectra of both components (see Fig. 5). We also confirm the presence of absorption lines due to $\mathrm{MgII}, \mathrm{MgI}$ and FeII in the spectra of Q1009-0252 A \& B at redshifts $z_{\mathrm{a}}=0.866$ and $z_{\mathrm{a}}=1.622$ (see Paper I). A more thorough analysis of these absorption line systems is presented by Hewett et al. on the basis of higher spectral-resolution and $\mathrm{S} / \mathrm{N}$ data. Note that the latter authors have detected the $z_{\mathrm{a}}=0.866$ absorption lines due to MgII in the spectrum of Q1009$0252 \mathrm{C}$; the spectrum of this quasar presented in Paper I has a too low $\mathrm{S} / \mathrm{N}$ to confirm this detection. Finally, when comparing the spectra from Fig. 5 with those published by Hewett et al., our spectrum of component B shows a systematic excess of light at wavelengths shorter than $4500 \AA$. It could be that time variability is responsible for these variations: our spectra were obtained at the beginning of the flux variation visible in the $B$ band in Fig. 3 while Hewett et al.'s observations took place in April 1993, i.e. after that flux variation.

\subsection{Analysis of the spectra}

Let $I_{\mathrm{A}}(\lambda)$ and $I_{\mathrm{B}}(\lambda)$ be the observed flux distributions characterizing the spectra of the $\mathrm{A}$ and $\mathrm{B}$ components, respectively. As suggested earlier in Paper I, the intensity ratio $I_{\mathrm{A}}(\lambda) / I_{\mathrm{B}}(\lambda)$ is not a constant but shows a systematic spectral dependence (see Fig. 6). Longwards from $\lambda \simeq 4800 \AA$, this ratio shows an almost linear decrease with wavelength. It is as if component A looks bluer than component $\mathrm{B}$, but at wavelengths shorter than $\lambda \simeq 4800 \AA$, the trend is opposite. Furthermore, absorption dips are conspicuously visible at the positions of the Ly $\alpha+$ NV, CIV and CIII] emission-lines. Such dips cannot be accounted for on the sole basis of relative dust extinc-

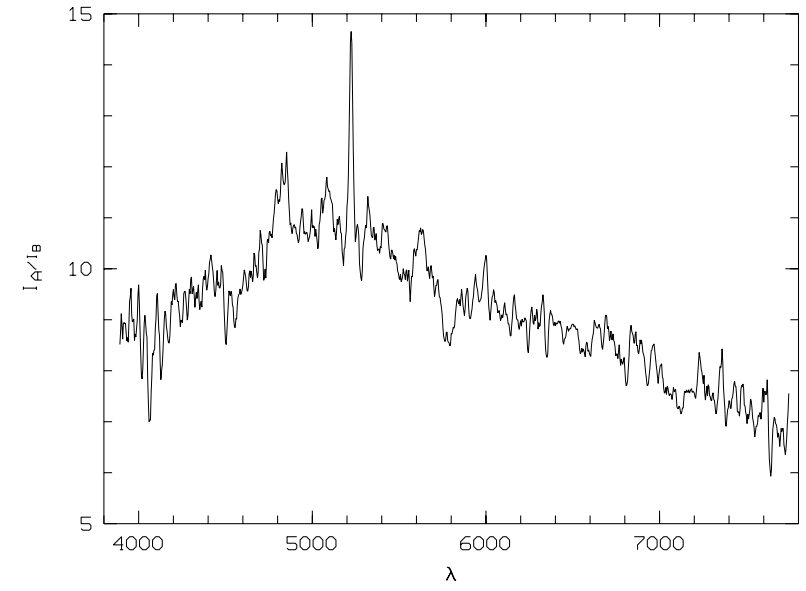

Fig. 6. Ratio between the spectra $I_{\mathrm{A}}(\lambda)$ and $I_{\mathrm{B}}(\lambda)$ as a function of wavelength

tion between the A and B components. Finally, the small emission peaks seen in Fig. 6 correspond to the locations of narrow absorption lines in the spectrum of $\mathrm{A}$ and $\mathrm{B}$, which are stronger in B than in A. This may easily be explained by differences in the optical depth of the corresponding absorbing gas clouds along the two lines-of-sight. Assuming that dust extinction correlates with the strength of these absorption lines, one would then naturally expect image $\mathrm{B}$ to be more reddened than image $\mathrm{A}$.

Although some observed spectral differences between images A and B could be induced by intrinsic spectral variations of the QSO combined with the expected time delay (Refsdal 1964), we first assume that they are only due to differential extinction effects (possibly arising in the lensing galaxy), macrolens and microlens amplifications. Thus, the observed flux distributions can be written as follows:

$\begin{aligned} I_{\mathrm{A}}(\lambda) & =\mathrm{e}^{-\tau_{\mathrm{A}}(\lambda)}\left[\mu_{\mathrm{A}}(\lambda) I_{0}^{c}(\lambda)+M_{\mathrm{A}} I_{0}^{e}(\lambda)\right] \\ I_{\mathrm{B}}(\lambda) & =\mathrm{e}^{-\tau_{\mathrm{B}}(\lambda)}\left[\mu_{\mathrm{B}}(\lambda) I_{0}^{c}(\lambda)+M_{\mathrm{B}} I_{0}^{e}(\lambda)\right]\end{aligned}$

The superscripts $c$ and $e$ stand for the continuum and emission contributions to the spectra, respectively, and the subscript 0 is used to specify the intrinsic QSO spectrum. The factors $\mathrm{e}^{-\tau_{\mathrm{A}}(\lambda)}$ and $\mathrm{e}^{-\tau_{\mathrm{B}}(\lambda)}$ represent the extinction affecting the $\mathrm{A}$ and $\mathrm{B}$ components, where $\tau_{i}(\lambda)$ quantifies the dust opacity along the line-of-sight to component $i$ as a function of wavelength. The emission lines in images $\mathrm{A}$ and $\mathrm{B}$ are only affected by the respective macrolens amplifications $M_{\mathrm{A}}$ and $M_{\mathrm{B}}$ while the continua are also possibly affected by the microlens amplification. The latter may show a smooth dependence with wavelength, depending on the relative size of the continuum source at different wavelengths with respect to the radius of the Einstein ring associated with the microlens(es) (for general reviews on microlensing, see Kayser et al. 1986; Nemiroff 1988, and Schneider \& Wambsganss 1990). The net amplification (micro+macro) of the continuum of image A (resp. image B) at wavelength $\lambda$ is called $\mu_{\mathrm{A}}(\lambda)$ $\left(\right.$ resp. $\left.\mu_{\mathrm{B}}(\lambda)\right)$. 


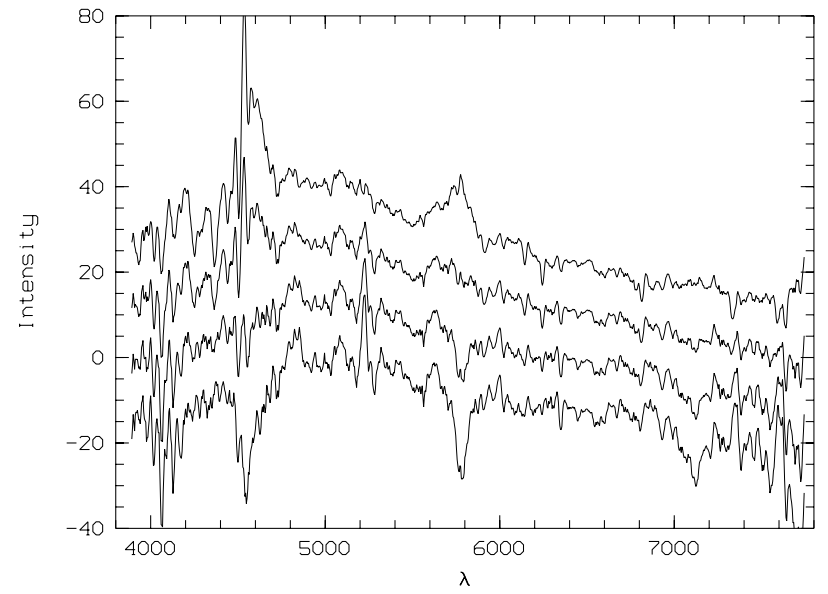

Fig. 7. Difference $I_{\mathrm{A}}(\lambda)-m I_{\mathrm{B}}(\lambda)$ as a function of wavelength for several values of the constant $m$. From top to bottom $m=$ $5,7,9,11$

Now, another way to combine the spectra of components $\mathrm{A}$ and $\mathrm{B}$ is to perform the difference $I_{\mathrm{A}}(\lambda)-m I_{\mathrm{B}}(\lambda)$, for different values of $m$ (see Fig. 7). If the spectra of components $\mathrm{A}$ and $\mathrm{B}$ were only differing by their macrolens amplification $M_{\mathrm{A}}$ and $M_{\mathrm{B}}$, the residual spectrum should be equal to zero for the particular value of $m=M=M_{\mathrm{A}} / M_{\mathrm{B}}$. However, no such zero residual spectrum is found. Moreover, the residuals systematically show a spectral dependence as well as features left over from a bad subtraction of the corresponding emission lines. The expression of this residual spectrum is:

$$
\begin{aligned}
& I_{\mathrm{A}}(\lambda)-m I_{\mathrm{B}}(\lambda)= \\
& \quad\left[\mathrm{e}^{-\tau_{\mathrm{A}}(\lambda)} \mu_{\mathrm{A}}(\lambda)-\mathrm{e}^{-\tau_{\mathrm{B}}(\lambda)} m \mu_{\mathrm{B}}(\lambda)\right] I_{0}^{c}(\lambda)+ \\
& {\left[\mathrm{e}^{-\tau_{\mathrm{A}}(\lambda)} M_{\mathrm{A}}-\mathrm{e}^{-\tau_{\mathrm{B}}(\lambda)} m M_{\mathrm{B}}\right] I_{0}^{e}(\lambda) .}
\end{aligned}
$$

According to this result, the residual spectrum will not show any sign of emission lines if the second term in Eq. (3) is null. This is always the case provided that

$m=m(\lambda)=\mathrm{e}^{-\Delta \tau(\lambda)} M$

with

$\Delta \tau(\lambda)=\tau_{\mathrm{A}}(\lambda)-\tau_{\mathrm{B}}(\lambda)$,

$M=M_{\mathrm{A}} / M_{\mathrm{B}}$

In order to estimate values of the function $m(\lambda)$ from the available spectra, let us consider once more Fig. 7 which shows the curves $I_{\mathrm{A}}(\lambda)-m I_{\mathrm{B}}(\lambda)$ with $m$ being a different constant along each curve. As it follows from Eqs. (3) and (4), it is possible to eliminate the signature of an emission line at a given wavelength $\lambda_{\mathrm{e}}$, if the constant $m=m\left(\lambda_{\mathrm{e}}\right)$. Figure 7 illustrates very well this statement for the three brightest emission lines: $m$ is taken to be approximately equal to 9,7 , and 5 for $\operatorname{Ly} \alpha(\lambda \approx 4600 \AA)$, $\operatorname{CIV}(\lambda \approx 5800 \AA)$ and $\mathrm{CIII}](\lambda \approx 7100 \AA)$, respectively. For this particular choice of the $m=m\left(\lambda_{\mathrm{e}}\right)$ values, we find that within the observed wavelength range the dependence of $m(\lambda)$ can be approximated by a linear function, namely

$m(\lambda)=16.67-0.00167 \lambda$.

We must admit that the above relation depends on the somewhat arbitrary choice of the acceptable $m=m\left(\lambda_{\mathrm{e}}\right)$ values. Nevertheless, it is now interesting to calculate the difference and the ratio of the spectra $I_{\mathrm{A}}(\lambda)$ and $I_{\mathrm{B}}^{\prime}(\lambda)=I_{\mathrm{B}}(\lambda) m(\lambda)$, where $I_{\mathrm{B}}^{\prime}(\lambda)$ represents the observed spectrum $I_{\mathrm{B}}(\lambda)$ corrected for differential extinction and relative macrolensing amplification of component $B$ with respect to $\mathrm{A}$. It is straightforward to derive the following results

$I_{\mathrm{A}}(\lambda)-m(\lambda) I_{\mathrm{B}}(\lambda)=\mathrm{e}^{-\tau_{\mathrm{A}}(\lambda)} \mu_{\mathrm{A}}[1-M / \mu] I_{0}^{c}(\lambda)$,

and

$\frac{I_{\mathrm{A}}(\lambda)}{m(\lambda) I_{\mathrm{B}}(\lambda)}=\frac{\frac{M_{\mathrm{A}}}{\mu_{\mathrm{A}}} I_{0}^{e}(\lambda)+I_{0}^{c}(\lambda)}{\frac{M_{\mathrm{A}}}{\mu_{\mathrm{A}}} I_{0}^{e}(\lambda)+\frac{M}{\mu} I_{0}^{c}(\lambda)}$,

where

$\mu=\mu_{\mathrm{A}} / \mu_{\mathrm{B}}$

represents the relative strength of the combined micro/macrolensing amplification of component A with respect to component B. In the absence of microlensing effects for both the $\mathrm{A}$ and $\mathrm{B}$ components, $\mu=M$ and, according to Eqs. (8) and (9), $I_{\mathrm{A}}(\lambda)-m(\lambda) I_{\mathrm{B}}(\lambda)=$ 0 and $I_{\mathrm{A}}(\lambda) /\left(m(\lambda) I_{\mathrm{B}}(\lambda)\right)=1$. We have illustrated in Figs. 8 and 9 the difference and the ratio between the spectra $I_{\mathrm{A}}(\lambda)$ and $I_{\mathrm{B}}^{\prime}(\lambda)=I_{\mathrm{B}}(\lambda) m(\lambda)$. We directly see that the residual spectrum in Fig. 8 differs from the null spectrum, although the residuals due to the emission lines have totally disappeared. The spectrum ratio in Fig. 9 shows a complex dependence with wavelength. If we concentrate on regions in the continuum, we may set $I_{0}^{e}(\lambda)=0$ in Eq. (9) and find that the spectrum ratio $I_{\mathrm{A}}(\lambda) / m(\lambda) I_{\mathrm{B}}(\lambda)=\mu / M$.

\subsection{Discussion}

One possible explanation for the derived spectral dependence of the ratio $\mu / M$ in the continuum seen in Fig. 9 is that microlensing amplification significantly affects component $\mathrm{A}$ and that the QSO source size strongly varies with wavelength, especially for $\lambda \leq 5000 \AA$. The trend of smaller equivalent widths observed for the emission lines in the $\mathrm{A}$ spectrum could also be accounted for by microlensing affecting the A component. However, the photometric lightcurves displayed in Fig. 3 are more compatible with a microlensing event affecting the $\mathrm{B}$ image than affecting the $A$ image. Therefore, although microlensing might well be at work, it cannot coherently explain all the observed anomalies between the A and B lensed images. We think that an intrinsic variation of the blue part of the spectrum cannot be avoided. The enhancement of the blue magnitude in the lightcurve of component B and 


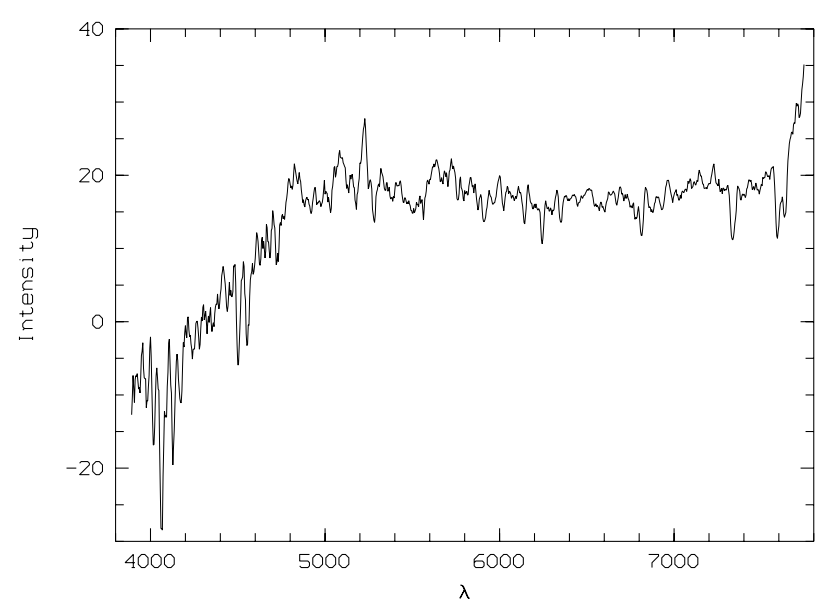

Fig. 8. Residual spectrum $I_{\mathrm{A}}(\lambda)-m(\lambda) I_{\mathrm{B}}(\lambda)$ as a function of wavelength (see text and Eq. (8))

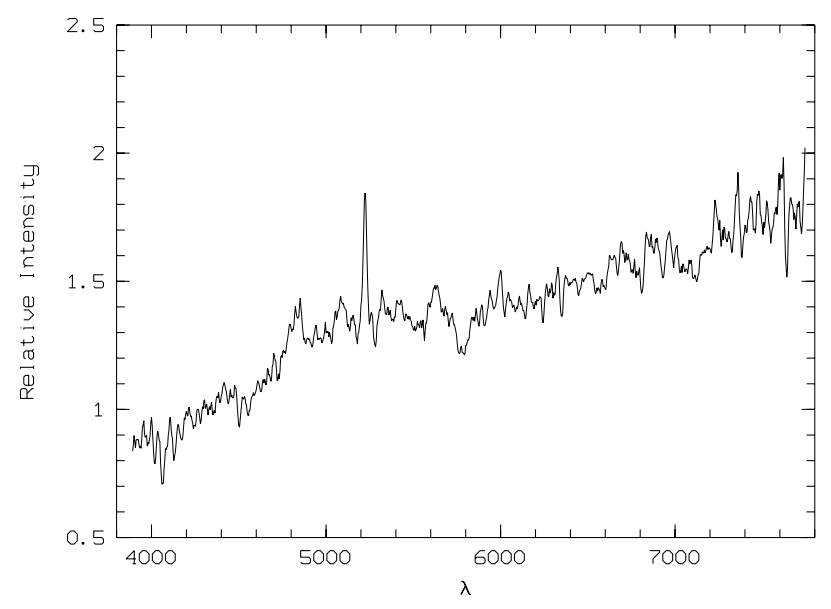

Fig. 9. Spectrum ratio $I_{\mathrm{A}}(\lambda) / m(\lambda) I_{\mathrm{B}}(\lambda)$ as a function of wavelength (see text and Eq. (9))

the "spectral break" blueward of $4800 \AA$ in the residual spectrum (see Fig. 8) would then be more naturally explained. Moreover, the response of the emission lines after a short time lag to the flux variation of the blue continuum could also affect their equivalent widths. Thus, although we have no definite conclusion, it seems that Q1009-025 A $\&$ B showed a complicated interplay of possible intrinsic flux variations and microlensing effects at the time of the observations. Since global variability does also show up in our HST observations, we warmly recommend (spectro)photometric monitoring of this gravitational lens. This would help in disentangling both effects and allow to determine the time delay and/or to perform microlensing studies.

Finally, note that according to Eq. (4), Eq. (7) shows the same spectral dependence as the differential extinction law $\exp [-\Delta \tau(\lambda)]$ of the lensing galaxy. However, due to the microlensing and/or intrinsic flux variations in the $B$ band, the extinction law cannot be fully retrieved. We can just say that the Milky-Way extinction curve [with $R_{V}=A(V) / E(B-V)=3.1$ for the standard value of the diffuse interstellar medium, Cardelli et al. 1989], red- shifted to the probable lens redshift $z_{1}=0.87$, is compatible with the spectroscopic data redward of $5000 \AA$ if the differential visual extinction $A_{\mathrm{V}}=0.95 \mathrm{mag}$ between the two lines of sight (cf. Eqs. (4) and (7)). Note that, assuming the $H$ band photometry is not affected by dust extinction, this value of $A_{\mathrm{V}}$ is also in full agreement with the differential reddening observed between the lensed QSO images in wide band photometry: $\Delta V-\Delta H=0.96$ (see Table 1 and Lehár et al. 2000). But the precise extinction law of dust present in the lens remains under-constrained by our data.

\section{Conclusions}

In this paper, we have analysed the properties of Q1009$0252 \mathrm{~A} \& \mathrm{~B}$ and of the lensing galaxy on the basis of new HST observations (with the WFPC2), new groundbased optical images, a new analysis of spectra presented by Surdej et al. (1993) and existing data from the literature.

The morphological study of the F814W images of the lens did not lead to definite conclusions but a pure de Vaucouleurs model with $r_{\mathrm{e}}=0.19^{\prime \prime}$ (Lehár et al. 2000) seems to be excluded by our data. Nevertheless, our optical photometry of the lens combined with the $H$ band photometry obtained by Lehár et al. (2000) shows that the lensing galaxy is very likely an elliptical at redshift $z_{1} \simeq 0.8$. Therefore, it could well be associated with the absorption line system identified at $z_{\mathrm{a}}=0.87$ (Paper I, Hewett et al. 1994, Sect. 4.3). Simple lensing models then provide a measurement of the mass-to-light ratio: $\left(\mathrm{M}_{\mathrm{E}} / \mathrm{L}_{\mathrm{E}}\right)_{I_{\mathrm{c}}}=4.5+0.5 h_{65}$. The lens induces a differential absorption of about $1 \mathrm{mag}$ in the $V$ band but a more precise study of the extinction law is not possible with the present data.

Multicolour lightcurves constructed from new and existing material indicate that Q1009-0252 is variable.

Although differential extinction and/or microlensing may help in accounting for some of the spectral differences observed between Q1009-0252 A \& B, these explanations are not entirely satisfactory. It could be that intrinsic spectral variability of the quasar coupled with the expected effects of time delay between the lensed A and B components is partly responsible for the differences observed in the profiles of the Ly $\alpha+\mathrm{NV}$, CIV and CIII] emission lines. A spectro-photometric monitoring of this intriguing gravitational lens system is absolutely mandatory in order to disentangle between the various possible causes discussed above. The measurement of the time delay and/or microlensing studies and a better determination of the extinction law in the lensing galaxy would then be possible.

Acknowledgements. We thank M. Fioc, who provided us with his code to compute the evolutive K-correction in different cosmologies, and Ch. Jean who helped us with the computation of the extinction. DWL would like to thank Olaf Wucknitz for helpful discussions. We should also like to thank the Belgian Office for Scientific, Technical, and Cultural Affairs for having 
given the possibility to SVKh to stay and work in Belgium during one year. Our research was supported in part by PRODEX (Gravitational lens studies with HST), by contract P4/05 "Pôle d'Attraction Interuniversitaire" (OSTC, Belgium) and by the "Fonds National de la Recherche Scientifique" (Belgium).

\section{Appendix: Inverting the lens equation}

The lens Eq. (1a) can be inverted for $\varphi=0$ (i.e. in the shear main axes). Indeed, with $\boldsymbol{\xi}=\left(\xi_{1}, \xi_{2}\right)$ and $\boldsymbol{\eta}=\left(\eta_{1}, \eta_{2}\right)$, and after some algebra, a fourth order polynomial equation is found for the coordinate $\xi_{1}$ of the images:

$a_{1} \xi_{1}^{4}+a_{2} \xi_{1}^{3}+a_{3} \xi_{1}^{2}+a_{4} \xi_{1}+a_{5}=0$

where

$a_{1}=4 \gamma^{2}(1-\gamma)^{2}$

$a_{2}=4 \eta_{1} \gamma(1-\gamma)(1-3 \gamma)$

$a_{3}=(1+\gamma(13 \gamma-10)) \eta_{1}^{2}+(1-\gamma)^{2} \eta_{2}^{2}-4 \gamma^{2} \theta_{\mathrm{E}}^{2}$

$a_{4}=-2 \eta_{1}\left((1-3 \gamma) \eta_{1}^{2}+(1-\gamma) \eta_{2}^{2}+2 \gamma \theta_{\mathrm{E}}^{2}\right)$

$a_{5}=\eta_{1}^{2}\left(\eta_{1}^{2}+\eta_{2}^{2}-\theta_{\mathrm{E}}^{2}\right)$.

Once the roots are found, either analytically or numerically, the coordinate $\xi_{2}$ of each image is found by the relation:

$\xi_{2}=\left(\eta_{2} \xi_{1}\right) /\left(2 \gamma \xi_{1}+\eta_{1}\right)$.

Finally, it is necessary to check the roots with the lens equation so as to find the real observable images.

\section{References}

Allen, C. W. 1976, Astrophysical Quantities, 3rd edition Cousins, A. W. J. 1976, Mem. R. astr. Soc., 81, 25

Cardelli, J. A., Clayton, G. C., \& Mathis, J. S. 1989, ApJ, 345, 245

de Vaucouleurs, G. 1948, Ann. Astrophys., 11, 247

Faber, S. M., \& Jackson, R. E. 1976, ApJ, 204, 668

Fioc, M., \& Rocca-Volmerange, B. 1997, A\&A, 326, 950
Freeman, K. C. 1970, ApJ, 160, 811

Fukugita, M., Shimasaku, K., \& Ichikawa, T. 1995, PASP, 107, 945

Hawkins, M. R. S. 1997, A\&A, 328, L25

Hewett, P. C., Irwin, M. J., Foltz, C. G., et al. 1994, AJ, 108, 1534

Horne, K. 1986, PASP, 98, 609

Kassiola, A., \& Kovner, I. 1993, ApJ, 417, 450

Kayser, R., Refsdal, S., \& Stabel, R. 1986, A\&A, 166, 36

Keeton, C. R., Kochanek, C. S., \& Falco, E. E. 1998, ApJ, 509, 561

Kormann, R., Schneider, P., \& Bartelmann, M. 1994, A\&A, 284,285

Krist, J., \& Hook, R. 1996, http://scivax.stsci.edu/ ${ }^{\sim} \mathrm{krist} /$ tinytim.html

Khmil, S. V., \& Surdej, J. 2000, A\&A, in preparation

Landolt, A. U. 1992, AJ, 104, 340

Lauer, T. R. 1985, ApJ, 292, 104

Lehár, J., Falco, E. E., Kochanek, C. S., et al. 2000, ApJ, 536, 584

Nemiroff, R. 1988, ApJ, 335, 593

Pahre, M. A., de Carvalho, R. R., \& Djorgovski, S. G. 1998, AJ, 116, 1606

Press, W. H., Flannery, B. F., Teukolsky, S. A., \& Vetterling, W. T. 1990, Numerical Recipes (Cambridge University Press)

Refsdal, S. 1964, MNRAS, 128, 307

Remy, M., Surdej, J., Baggett, S., \& Wiggs, M. 1997, in The 1997 HST Calibration Workshop with a New Generation of Instruments, ed. S. Casarteno et al., 374

Remy, M., Claeskens, J.-F., Surdej, J., et al. 1998, New Astr., 3,379

Schmidtke, P. C., Windhorst, R. A., Mutz, S. B., et al. 1997, AJ, 113, 569

Schneider, P., \& Wambsganss, J. 1990, A\&A, 237, 42

Schneider, P., Ehlers, J., \& Falco, E. E. 1992, Gravitational Lenses, A\&A Library, ed. M. Harwitt, R. Kippenhahn, V. Trimble, \& J.-P. Zahn (Springer-Verlag)

Surdej, J., Remy, M., Smette, A., et al. 1993, in Gravitational Lenses in the Universe, ed. J. Surdej, D. Fraipont-Caro, E. Gosset, S. Refsdal, \& M. Remy, Université de Liège, 153 (Paper I)

Whitmore, B. 1997, http://www.stsci.edu/ftp/instrument_news/WFPC2/ Wfpc2_phot/photom2.ps

Witt, H., Mao, S., \& Keeton, C. R. 2000, ApJ, 544, 98 